\title{
CLASSIFICATION OF ACOUSTO-OPTIC \\ CORRELATION SIGNATURES OF \\ SPREAD \\ SPECTRUM SIGNALS USING ARTIFICIAL NEURAL NETWORKS
}

\author{
John W. DeBerry David M. Norman \\ Air Force Institute of Technology ( $\mathrm{AU}$ ) \\ Wright-Patterson AFB, OH
}

\begin{abstract}
The primary goal of this research was to determine if Artificial Neural Networks (ANNs) can be trained to classify the correlation signatures of direct sequence and frequency-hopped spread-spectrum signals. Secondary goals were to determine (1) if network classification performance can be modeled with a conditional probability matrix, (2) if the symmetry of the matrices can be controlled, and (3) if using a majority vote rule over independently trained networks improves classification performance. Correlation signatures of the spread-spectrum signals were obtained from United States Army Harry Diamond Laboratories. The signatures were preprocessed and separated into various training and testing data sets. Thirty samples of network responses for several sets of training conditions were gathered using a neural network simulator. ANNs trained directly on correlation signature data yielded classification accuracies on test data at or near $80 \%$. The probability matrices were stationary with regard to test sets and the ability to shift the symmetry of the matrices was demonstrated. Improvement of classification accuracy via majority vote was possible if the nets were trained on different data sets. An average improvement of $1.8 \%$ was found to be statistically significant for $\alpha=0.05$. A metric was developed to estimate the similarity of the solutions found by networks in a given training run.
\end{abstract}

\section{Introduction}

Over the past two decades, use of spread-spectrum systems in the Department of Defense has become increasingly common. This is primarily due to the militarily desirable antijam $(\mathrm{AJ})$, antiinterference, and low probability of intercept (LPI) characteristics of these signals [1]. It is reasonable to assume that our adversaries will use spread-spectrum systems in any future conflict. Naturally, it follows that we should prepare to defeat the AJ and LPI characteristics of these signals in order to deny our enemy the free use of the electromagnetic spectrum.

In order to disrupt a hostile communications signal, we must be able to detect and classify the signal. Much of the current research is focused on solving this problem. Toward that end, researchers at the U.S. Army Harry Diamond Laboratories have developed a one-dimensional time-integrating acousto-optic (AO) correlator capable of $10^{15}$ operations per second [2] The device performs the correlation transform several orders of magnitude faster than even the most powerful digital computers. This correlator can be used in an intercept receiver to detect and capture the correlation signatures of spread-spectrum signals. Currently, the output of the correlator is examined directly by a human operator or digitized and run through curve-fitting routines on digital computers. The objective is to recognize the correlation signature and obtain information about its time domain modulation characteristics.

Researchers at U.S. Army Harry Diamond Laboratories have suggested using Artificial Neural Networks (ANNs) to classify spread-spectrum communications signals. The ANN would be trained to recognize and classify specific input features present in the correlation signatures of several types of spread-spectrum signals. These features correspond to time domain characteristics of the signal. Classification of the signals in this manner essentially extracts information about the modulation parameters used to construct the signal. In an eventual hardware implementation, the network would operate in real time at the output of an AO correlator. A first step in this effort would be to construct and train an ANN to determine whether a particular input spread-spectrum correlation signature is a direct sequence (DS) or frequency-hopped (FH) signal. The primary objective of this research is to answer several questions:

1. Can ANNs be trained to classify DS and FH correlation signatures, and if so, at what level of classification performance?
2. Can a trained ANN's response to previously unseen signatures be accurately modeled and described by a transition probability matrix similar to those used to describe communication channels? If so, can the symmetry of these matrices be controlled? Control of matrix symmetry would allow network classification responses to be tailored for a particular application.

3. Can classification accuracy be improved by using a majority vote decision rule over the response of an appropriate number of ANNs trained to classify the same correlation signatures?

\section{Methodology}

This section provides information concerning the details of how the experiments were performed. First, a description of the resources used is presented. This is followed by a description of how each of the experiments was designed and implemented. Finally, the manner in which the results of the exeriments were analyzed is given.

\section{Resources.}

The following paragraphs describe the spread-spectrum correlation product data files, the artificial neural network (ANN) simulator, and the preprocessing performed on the correlation product data in order to use it with the simulator.

The Correlation Product Data.

The spread-spectrum correlation signatures were generated by simulating various direct sequence (DS) and frequency hopped (FH) signals and feeding them into an acousto-optic $(A O)$ correlator. Different signatures of each type of signal were generated by varying several modulation parameters; chip rate, carrier frequency, psuedo-random code, etc. The FH signals were not driven by a psuedo-random code, but were stepped across frequency ranges by a linear stepper. For the remainder of this paper, exemplars derived from DS signatures will be known as class 1 exemplars, while those derived from FH signatures will be class 2 exemplars. The output of the AO correlator for a given signature was sampled and written to an ASCII file as a column of numbers. The files all contained 1,000 data points.

\section{The ANN Simulator.}

The ANN simulator used was the NeuralGraphics simulator written by Captain Greg Tarr [3]. Two minor modifications were made to support the data requirements of the experiments. First, a terminal test routine was added. The routine writes the network response to the test data set to a file. The file contains a test exemplar label, the true class, and the network's classification decision for each exemplar in the test data set. In addition, a training history file for the networks was also written to a file The history files contain the outcome of performance tests after every 1,000 training iterations until training was terminated. The second modification to the software concerned the setting of the seeds for the random number generator. The generator is used to select values for the initial weight values of the nets at the start of training and to randomly select an exemplar for presentation to the net during training. These training conditions were controlled by varying how and when the generator seed was selected. For example, when it was desired that successive nets be trained from the same initial weight state, the seed for selecting the initial weights was set to the same arbitrary constant for each net trained. If it was desired that successive nets be trained from different initial weight states, the seed was set to the current value of the system real time clock. Control over the order of presentation of training exemplars was obtained in a similar manner.

The NeuralGraphics simulator allows the user to select one of several network structures and training algorithms. The back propagation net-

U.S. Government work not protected by U.S. copyright. 446 
works all use the update rules described [4]. The values of the learning rate $\eta$ was 0.3 , while the momentum gain $\alpha$ was 0.8 . Preliminary training runs showed that the networks trained relatively well over a wide range of combinations and numbers of hidden layer nodes. Of the various combinations tried, ten nodes in the second hidden layer and 18 nodes in the first hidden layer appeared to yield the most consistent classification performance. Also, it was observed that no improvement in performance occurred af ter 20,000 training iterations, therefore this was used as the criterion to terminate training.

\section{Data Set Construction.}

The number of output nodes and input nodes of a network is determined by number of classes to be recognized and the number of elements in the input exemplar. Since presentation of all 1,000 elements of a data file as inputs to a network was beyond the capacity of the simulation software, all of the data files containing the correlation signatures were processed in the following manner. First, the data files were reduced to 500 points by averaging consecutive pairs of data points together. Next, the data point with the largest absolute value was found and all data points divided by this value. The result of these two steps was a 500 point signature pattern linearly compressed to values between -1 and 1 . The final step was to extract a 50 point window centered about the peak positive value of the correlation product pattern. A total of 101 class 1 and 108 class 2 exemplar patterns were available to train the networks. The data sets used by the simulator to train networks for the different experiments were constructed from this pool of exemplars.

\section{Definitions and Notation.}

Before proceeding with the descriptions of the experiments, the terms and notation used in the remainder of this paper must be defined. There are several metrics used by the NeuralGraphics simulator to evaluate network performance: total error, right classification, and good classification. The network yields a right classification if the error at each output node, for that exemplar, is less than 0.2 . A good classification only requires that the maximum output occur at the node representing the class of the input cxemplar [3]. For example, if the desired output for a given exemplar were $[1,0,0]$, then $[0.91,0.09,0.09]$ would be a right classification and $[0.7$ $0.5,0.4$ ] would be a good classification. The focus of this research was to examine the effect of the training conditions on only the good classification performance on test data sets. Furthermore, this metric will be viewed in probabilistic terms. For example, assume a certain trained network is tested with a data set having 50 class 1 exemplars and 50 class 2 exemplars. If the network yields a good classification for 80 of the 100 test exemplars, this will be expressed as $P$ (good) $=0.80$. Also, if the network yields good classification on 35 of the 50 class 1 exemplars and 45 of the 50 class 2 exemplars in the test data set, then these observations will be expressed as the conditional probabilities, $P(1 \mid 1)=0.70$ and $P(2 \mid 2)=0.90$. It should be clear that conditional probabilities for incorrect classification are $P(2 \mid 1)=1-P(1 \mid 1)$ and $P(1 \mid 2)=1-P(2 \mid 2)$. For this example the proportions of the class 1 and class 2 exemplars in the test set can be expressed as the a priori probabilities $P(1)=P(2)=0.50$.

The characterization of network performance in this manner is similar to the way information channels are characterized in the communications field. The conditional probabilities are known as channel transition probabilities and collectively referred to as the transition probability matrix or $\underline{P}$ matrix. Networks trained for a two class recognition problem are modeled as a binary channel. For further information regarding channel models, the text by Hamming [5] is an excellent choice. Characterization of network performance with this model was inspired by the confusion matrix found in the work of Piazza [6]. These confusion matrices were simply counts of how the input exemplars of each class were distributed over the outpu classifications by the network. If the number of test exemplars is sufficiently large, it is not hard to extend the confusion matrix concept to the $P$ matrix described above.

Finally, a few words regarding the naming conventions used are in order. Since there are random processes involved in training networks, the value of the $P$ (good) is a random variable with some probability density function (PDF), and it will have some distribution about a mean. Examination of the effects of different training conditions or test conditions must be done by examining the differences in the distributions of $P$ (good). Samples of these distributions, or PDFs, were obtained by training a number of networks for a given set of conditions and observing the outcomes. In the next section, a total of five sets of training conditions will be specified. In addition to this, there will be three different methods for generating distributions of interest. Specifically, the $P(1 \mid 1), P(2 \mid 2)$, and $P$ (good) distributions for
(1) observed outcomes of individual networks, (2) outcomes of majority vote networks constructed from observed outcomes of three individual networks, and (3) the calculated outcomes of majority vote nets constructed using the probability matrices of three individual nets. As one can see, quite a number of different PDFs will need to be named and referred to. The following shorthand naming conventions shall be used. The letter $R$ followed by a single digit will specify a set of training conditions for a training run. The letter $S$ will indicate the distribution is for single nets. The letter $M$ will signify the distribution is for majority vote nets. The appearance of P11, P22, or PG will designate the distributions as $P(1 \mid 1), P(2 \mid 2)$, or $P$ (good), respectively. A letter $C$ prepended to the name indicates a calculated distribution. As an example, the string R1SP11 refers to the $P(1 \mid 1)$ distribution for the single nets of Run 1 . Similarly, the string CR5MPG refers to the calculated $P$ (good) distribution for majority vote networks of Run 5 .

\section{Experiment Design.}

All of the experiments defined in this section are designed to examine the influence that various conditions have on the good classification performance metric. For each experiment, the purpose or intent, network training and testing conditions, and expected results will be specified. Additionally, the data requirements and distribution nomenclatures will also be given. Table 8 summarizes the conditions for training and testing the networks of each experiment.

\section{Training Performance.}

While not an actual experiment in the true sense of the word, the documentation of how the networks train to their final states is of general interest. It can guide future research using the same data sets by documenting a baseline performance for comparisons. The good classification performance was averaged over 30 nets trained in a given run, at 1,000 iteration intervals. These averages were plotted against iterations to yield a training performance curve.

Characterization of ANN Performance with the P Matrix Model.

In this experiment, the validity of using a $P$ matrix to characterize the performance of trained networks was tested. Specifically, the stationarity of the matrix over different test data sets for networks trained in the same manner, was examined. Thirty networks were trained using the same 102 training exemplars. The initial weights at the start of training and the presentation order of training exemplars were different for each net trained. These networks were tested with a baseline data set having 50 class 1 exemplars and 50 class 2 exemplars. The same networks were also tested with data sets having a $40 / 60 \%$ mix of class 1 to class 2 exemplars. Thirty of these data sets were constructed from the baseline test set by randomly removing 12 class 1 exemplars and adding seven class 2 exemplars. The results of testing the 30 nets with the baseline test set will be called Run 1 , while the results of testing the 30 nets with data sets having the $40 / 60 \%$ exemplar mix will be called Run la.

A total of seven distributions were needed to perform the experiment. The distributions for the conditional probabilities, $P(1 \mid 1)$ and $P(2 \mid 2)$, and the joint probability, $P$ (good), were generated for each run. In addition, a calculated $P$ (good) distribution was generated by using the $P$ matrices of Run 1 and assuming the a priori probabilities $P(1)=0.40$ and $P(2)=$ 0.60 . The nomenclatures for these distributions are; R1P11, R1P22, R1PG, CR1PG, R1AP11, R1AP22, and R1APG.

If the $\underline{P}$ matrices are stationary with respect to test data sets, then the following results should be expected. There should be no difference between the $P(1 \mid 1)$ and $P(2 \mid 2)$ distributions of either run, nor should there be any difference between the calculated $P$ (good) distribution of Run 1 and the $P$ (good) of Run 1a. The difference between the $P$ (good) PDFs of Run 1 and Run 1a should be due only to the change in the a priori probabilities of the exemplar classes in the test data sets.

\section{Controlling $P$ Matrix Symmetry.}

From the preliminary training runs, it was known that the conditional matrices for nets trained with a $50 / 50 \%$ mix of exemplar classes were asymmetric. The good classification of class 1 exemplars was much poorer than for class 2 exemplars. If this $\underline{P}$ matrix were for a communications channel, adjustments would be made so that the channel was symmetric $[5,143]$. Assuming it would be desirable to have a neural network with a symmetrical $P$ matrix, a question arises: Is it possible to cause the $P$ matrix to move toward symmetry? This experiment was designed to answer this question.

The $P$ matrix PDFs of Run 1 will be used as the baseline case of trained 
network responses. Another run of nets, which shall be referred to as Run $2 \mathrm{~S}$, was trained with a $60 / 40 \% \mathrm{mix}$ of class 1 to class 2 exemplars. The training sets were constructed by randomly removing 17 class 2 exemplars from the baseline training set of Run 1. The test set exemplars for all of these nets were identical to the test set used for Run 1 . The three new PDFs of interest are the $P(1 \mid 1), P(2 \mid 2)$, and $P$ (good) of Run $2 S$ which will have the nomenclatures R2SP11, R2SP22, and R2SPG.

If the networks are trained harder on class 1 exemplars, as in Run $2 S$, then the weight space solutions found by the nets should shift to be more favorable to class 1 recognition. In terms of the PDFs, the mean value of the $P(1 \mid 1)$ PDF in Run 2 should be greater than that of Run 1, and the mean of the $P(2 \mid 2)$ PDF of Run 2 should be less than that of Run 1. It is hoped that the means of the two $P$ (good) distributions are essentially the same.

Improvement of Classification Performance via Majority Vote Rule.

This experiment was designed to determine if classification performance can be improved by using a majority vote decision rule over three separately trained nets. In addition, the conditions of training necessary to achieve this improvement was explored. The idea proposed here is very similar to the use of redundancy in communications systems to reduce probability of syrnbol or bit error. Basically, for a channel having a specified probability of bit error, two redundant bits are sent for every information bit. If the cause of corruption (noise) is independent and uncorrelated during each successive bit transmission, then the joint probability that two or all three bits are in error will be less than that of any single bit. However, in order to realize the improvement, there must be independence from one trial to the next. It is obvious that the solutions found by the successively trained networks are not totally independent; the back propagation algorithm is seeking the same global minimum on the same error surface on each trial. However, one cannot say that the solutions are totally dependent either since it has been observed that the nets do not end up in exactly the same place in weight space [6, page 4-9].

Four runs of nets trained under different conditions were required for this experiment. The test data sets for these four runs were identical. Run $2 S$ nets were trained such that initial weights, presentation order of training exemplars, and exact composition of the training data set were varied from one net to the next. An additional 90 nets were trained in this manner These nets were then used three at a time to construct 30 majority vote nets. The $P$ matrices for these nets were generated by observing the decisions of each of the single nets for the test set exemplars and determining the final classification by majority vote. At the same time, a calculated majority vote matrix was generated by assuming independence and using the $P$ matrices of the the 90 single nets three at a time. This same basic procedure was repeated for runs 3,4 , and 5 : 120 nets were trained, 30 nets were used to construct the single net PDFs, the other 90 to construct the majority vote and calculated majority vote PDFs. The nets of Run 3 were all trained on the same data set, but the initial weights and exemplar presentation order were different for every net. In Runs 4 and 5 , the training data sets were the same as Run 3, but in Run 4, only the initial weights varied, while in Run 5, only the presentation order varied. The nomenclatures for the PDFs to be compared are: R2SPG, R2MPG, CR2MPG, R3SPG, R3MPG, CR3MPG, R4SPG, R4MPG, CR4MPG, R5SPG, R5MPG, and Cl.5MPG.

If there is a sufficient degree of independence in the outcomes of the single nets of any of the runs, the result should be that the average of the majority vote $P$ (good) PDF is greater than that of the single nets. This gain in average performance should be some portion of the gain for the calculated majority vote nets. Also, comparisons between the majority vote gains of the different runs should provide some insight into the relative degree each controlled condition contributes to the randomness of the weight space solutions found by the nets.

Influence of Training Data Sets, Initial Weights, and Exemplar Presentation Order on Network Solutions.

This experiment uses the data obtained while training the nets of runs 2 through 5. The intent of this experiment was to discover the degree of similarity of decision regions formed by nets in a given run.

For each network trained in the previous experiment, a list of the exact exemplars incorrectly classified by that net was generated. The lists for the first 30 nets trained in each run were compiled into a master list for each run. The list contains the file name of all the exemplars incorrectly classified by any of the 30 nets in a given run. In addition, the exact numbe of nets in the run that incorrectly classified each exemplar on the list wa recorded.
If the decision regions formed by the nets of a given run are all relatively similar then it a majority of the test exemplars on the list will be incorrectly classified by most, if not all, of the networks trained for that run. In other words, if two nets find exactly the same solution, they should make exactly the same mistakes. On the other hand, if two nets find equally good, but very different decision regions, then even though each net makes the same number of mistakes, the mistakes made by one may be quite different than those made by the other.

\section{Analytical Methods.}

The quantities being dealt with in this thesis are samples of populations. In some cases, the samples may in fact be from the same population and in other cases, from entirely different populations. By analysis and comparison, we hope to discover which of these statements apply to the sample distributions being compared. The method chosen to do this is hypothesis testing. The PDFs were tested using a commercial software package, Statistix $^{t m}$, from NH Analytical Software [7], running on an IBM compatible personal computer. For all comparisons equality is the standard null hypothesis. Alternative hypotheses may be inequality, less than, or greater than, as it fits the particular comparison. Additionally, the standard level of significance for all tests will be $\alpha=0.05$. The software package provides the p-value for all tests. A p-value is the probability that the observed difference in the samples could have occurred by random chance. Thus, a very low p-value indicates that the samples are not from the same population. If the $p$-value falls below the $\alpha$ value, the null hypothesis is rejected, and one may accept the applicable alternative hypothesis.

There are many test statistics available for hypothesis testing. The parametric tests are, in general, more powerful, but require that the samples come from a normal population. Non-parametric tests only require that the observations be independent. As a standard procedure, a test for normality using the Wilk-Shapiro test statistic [8] was performed. Whenever the assumption of normality held, a parametric Two-Sample t-test was performed, otherwise, non-parametric tests were used. For further information on hypothesis testing or test statistics, refer to [7] [9] [10]

\section{Results}

\section{Average Good Classification Histories on Test Data.}

Figure 1 shows the histories of percent good classification on test data Run 3 , which was used as a baseline, and was typical of all the training runs. The figure has plots of the average value, the average plus one standard deviation, and the average minus one standard deviation. After 1,000 iterations, the nets show close to $70 \%$ classification accuracy. A point at 10,000 iterations was well past the knee of the curve and at 20,000 the net attains roughly $80 \%$ classification. The standard deviation approaches a constant value of 1.604 .

Characterization of ANN Performance with the $P$ Matrix Model.

Table 2 shows the average and standard deviation of the PDFs of interest. Additionally, the difference in the average values are shown in the last row of the table. The table shows that the largest difference is between the observed joint PDFs, $P$ (good), of Run $1 \mathrm{~A}$ and Run 1 , while the smallest difference was between the observed $P$ (good) PDF of Run $1 \mathrm{~A}$ and the calculated $P$ (good) PDF of Run 1.

Table 3 shows the results of testing the null hypothesis that the sample distributions being compared are actually samples from the same population. Note that there is a failure to reject the hypothesis for the comparisons between the two $P(1 \mid 1)$ PDFs and between the calculated $P$ (good) of Run 1 and the observed $P$ (good) of Run $1 \mathrm{~A}$. There is also a unanimous agreement between the tests to strongly reject the hypothesis for Run $1 \mathrm{~A}$ and Run $1 P$ (good) distributions. Unfortunately, there is a conflict in the decision about the comparison of the $P(2 \mid 2)$ PDFs of the two runs. Three of the four tests dictate a rejection of the null hypothesis. It was expected that there would be a failure to reject the null hypothesis in this comparison.

The results show no distinguishable difference between the conditional $P(1 \mid 1)$ distributions of the two runs or the observed joint distribution of Run $1 \mathrm{~A}$ and the calculated joint distribution of Run 1. The tests show strong evidence that the two $P(2 \mid 2)$ PDFs are from different populations, which is exactly opposite of what was expected, even though these PDFs had onc of the smallest differences between their averages. However, the sum of their standard deviations was also the smallest. When the variances of two samples are small, then minute differences in their means can be detected by statistical tests. 


\section{Controlling $\underline{P}$ Matrix Symmetry.}

In Table 4, the averages and standard deviations of the PDFs of Run 1 and Run $2 S$ are shown. The last row of the table shows the difference in the average values of the like PDFs of each run. In all three instances, the differences are fairly large. The reader should note that the $P$ matrix of Run 1 is skewed in favor of the $P(2 \mid 2)$ PDF while in Run $2 S$ the averages of $P(1 \mid 1)$ and $P(2 \mid 2)$ PDFs are closer together and the average matrix is almost symmetric.

Table 5 shows the results of non-parametric hypothesis tests. In every case, there is unanimous agreement for strong rejection of this hypothesis. The tests indicate that it is highly unlikely that any of the distributions compared actually came from the same populations.

Improvement of Classification Performance via Majority Vote Rule.

In Table 6, the averages of the $P$ (good) PDFs generated from the runs are shown. The first column indicates the run to which the row applies. For the other column headers, $\mathrm{S}$ stands for single nets, $\mathrm{M}$ for observed majority vote, and $\mathrm{CM}$ for calculated majority vote. In all cases, the differences between means of the calculated majority vote PDFs and the single net PDFs was substantial. These differences should be viewed as the maximum expected improvement. Thus, if we calculate $(M-S / C M-S) \times 100$ we have a measure of how close to this maximum the observed values were. These values are approximately $19.7 \%, 7.0 \%, 1 \%$, and $-2.6 \%$ for runs 2 through 5 , respectively.

Table 7 shows the results of the tests of significance tests for comparisons between the PDFs of singles nets and majority vote nets within each run. In cvery case, there is unanimous agreement for strong rejection of the hypothesis for the comparison between Run 2 single nets and Run 2 observed majority vote nets. For all others runs, there is unanimous agreement to fail to reject the hypothesis. Although not shown in the table, the same tests were performed for the differences between the PDFs of single nets and calculated majority vote nets. They were not included in the Table 7 because the results can be stated rather simply; out to four decimal places, all of the $p$ values were zero. This same result applies to a comparison between the observed and calculated majority vote PDFs.

These results show that the difference between the means of the $P$ (good) distributions for single nets and observed majority vote nets of Run 2 was significant. The difference amounts to approximately $19.7 \%$ of the maximum expected improvement. Conversely, the observed difference in these PDFs for all other runs was not significant.

Influence of Training Data Sets, Initial Weights, and Exemplar Presentation Order on Network Solutions.

In order to gain insight into the differences between the networks of runs 2 through 5 , we present here an analysis of which test exemplars were incorrectly classified in each run. Also, the number of nets that incorrectly classified each of these exemplars is shown.

Table 8 contains the complete list of incorrectly classified exemplars along with a count of how many nets misclassified each exemplar. There were 54 exemplars on the list for Run 2, 34 for Run 3, and 30 for Runs 4 and 5 . The maximum number of incorrect classifications is 30 , since there were 30 nets trained in each run. The lists are ordered from highest to lowest number of error counts.

Note that several exemplars appear in all four lists; corr96, corr 25 , corr 194. One might suspect that these exemplars were abnormal or perhaps corrupted, and should be discarded. However, a visual inspection of the graphs of these data files failed to reveal any evidence that this was the case. A comparison of all the exemplars in the lists of Table 8 revealed that 23 exemplars were common to all four lists.

It should be evident by now that even when nets are trained with identical training sets, as in runs 3 through 5 , the solutions found by each net are not all equivalent. Even if two different nets classify 20 test exemplars incorrectly, those exemplars are not necessarily the same. All that is known is that the two solutions are equivalent with respect to classification performance. What is needed is a measure of the similarity of the decision regions formed by the networks.

For the purpose of illustration, assume that several nets trained for a run have all found exactly the same solution. Clearly, if the decision region boundaries are the same, each net would incorrectly classify exactly the same exemplars and the error count for each one is equal to the number of nets trained in the run. There is a maximum correlation between the errors in classification made by the nets. Define $N$ as the number of networks trained in the run, and $E$ as the number of different exemplars on the error list. Let $C_{i}$ be the the error count for the $i$ th exemplar on the list. It should be clear that, in this case

$$
\sum_{i=1}^{E} C_{i} / N E=1
$$

Now assume the other extreme. Assume each of the $N$ nets misclassifies only one test set exemplar, but each net misses a different exemplar. The error list now contains $E=N$ exemplars, each having an error count of 1 . For this situation,

$$
\sum_{i=1}^{E} C_{i} / N E=1 / N
$$

This suggests a metric, let it be called $L$, of the form

$$
L=\sum_{i=1}^{E} C_{i} / N E
$$

which is bounded by 1 , for maximum correlation in the error list, and $1 / N$, for minimum correlation. Note that if $N$ approaches infinity, then in the case of minimum correlation, $L$ could approach zero.

Applying this metric to the lists in Table 8 the values of $L$ are approximately $0.362,0.546,0.606$, and 0.667 for runs $2,3,4$, and 5 , respectively. Since, $L$ is a measure of the similarity of the decision regions formed by the nets of a given run, one would expect runs with lower $L$ values to yield the greater classification improvement under a majority vote rule than runs with higher $L$ values. Table 7 shows that this is true, with the exception of Run 5 .

Figures 2 through 5 are graphical representations of the counts in Table 8. Although the graphs do not show the exemplar labels, there is a one to one correspondence between the columns of the respective graphs and the entries in Table 8 (i.e. column 1 of Figure 2 is the value of the count for the first entry in the list for Run 2).

The figures show, in an intuitive way, the reasons for the results presented in the previous section. Clearly, if one arbitrarily chooses any three nets from a given run, the exemplars having error counts lower than 15 (i.e. less than a 0.5 probability of being incorrectly classified in that run) have a greater than 0.5 probability of being correctly classified under a majority vote rule taken over the three nets. Observe in Figure 2, that Run 2 has 38 exemplars, approximately $70 \%$, which fall into this category. For Runs 3 4 , and 5 , the proportions of exemplars in this category are approximately $44 \%, 40 \%$, and $33 \%$, respectively. Based on these proportions, it could be predicted, for a majority vote rule over three nets, that Run 2 nets would yield the greatest improvement in classification performance, Run 3 the next greatest, Run 4 the next, and finally Run 5 . The results presented in Table 6 support this prediction.

\section{Conclusions.}

The reader should be aware that the conclusions presented here only apply to the two class recognition problem for the data sets used in this study. It would be premature to apply these conclusions to ANNs trained on other data sets or even a three class problem using the same type of data sets.

\section{Training Performance.}

A three-layer back propagation neural network can train directly on correlation signatures of direct sequence (DS) and linearly stepped frequency hopped $(\mathrm{FH})$ spread spectrum signals. The networks can be expected to yield at or near $80 \%$ accuracy on test data sets after 10,000 training iterations. Additionally, the overall standard deviation of test set classification accuracy can be expected to be less than $2 \%$. It is clear from the plot shown in Figure 1 that by 10,000 iterations, the nets were at or near their maximum values for any of the classification performance metrics. Additional training past 10,000 iterations provided little, if any, improvement in test set classification accuracy. Additionally, no reduction in the variance was observed.

Characterization of ANN Performance with the $\underline{P}$ Matrix Model.

The $P$ matrix model is a valid and useful tool for describing and evaluating ANN classification performance. It was shown that the $P$ matrix obtained from testing 30 networks on one test data set accurately predicted the joint PDF obtained by testing the nets with data sets having a different proportions of exemplars. There was essentially no difference in the $\underline{P}$ 
matrices obtained from testing the nets with either test data set. While this statement is true for the conditional PDFs of class 1 exemplars, it may be debatable for the PDFs of class 2 exemplars. However, it was observed that the nets did very well at recognizing class 2 exemplars and the PDFs had much smaller variances. This small variance caused three of four tests of significance to reject the null hypothesis that the distributions were the same, even though there was a relatively small difference in their means. Thus, it may be argued, from a practical viewpoint, that there was actually no difference between these PDFs. The implication of these observations is that the responses of the networks were stationary. The change observed in the average joint $P$ (good) PDF was due only to the change in the a prior probabilities $P(1)$ and $P(2)$. Thus, the conditional $P$ matrix is a more useful description of a network's classification performance than the joint probability, $P$ (good)

\section{Controlling $\underline{P}$ Matrix Symmetry.}

Given that training on a particular data set does not yield the desired conditional classification performances, it is possible to change this by appropriate adjustments in the proportions of exemplar classes in the training data set. The average conditional probabilities, $P(1 \mid 1)$ and $P(2 \mid 2)$, for a particular run of 30 nets to be 0.7380 and 0.9213 . The average joint recognition probability was 0.8297 . Obviously, these nets were not recognizing class 1 exemplars as well as class 2 exemplars. The training data set proportions were changed from a $50 / 50 \%$ mix to a $60 / 40 \%$ mix of class 1 to class 2 exemplars and another run of 30 nets were trained. The resulting average conditional probabilities, $P(1 \mid 1)$ and $P(2 \mid 2)$ were 0.7767 and 0.8320 , while the joint probability was 0.8043 . Statistical tests on the PDFs confirmed that the changes were significant. The $P$ matrix was indeed adjusted toward symmetry. Clearly, this demonstrates a technique for controlling the symmetry of ANN classification behavior. However, the trade-off was a reduction in the joint classification accuracy. This was no too surprising because back propagation of error seeks to minimize the error over all training exemplars. If the training data set is weighted heavily toward one exemplar class, then clearly, the solution in the weight space will shift to favor that class since it now has a greater contribution to the overall error. Of course, the exact proportions needed to achieve symmetry would be dependent on the relative contribution to the overall error of each class of exemplars taken as a group. While the experimental objective was to adjust the $\underline{P}$ matrix toward symmetry, this may not be desirable for all cases. The point is that a network's classification response can be adjusted to whatever symmetry is best for a given application.

Improvement of Classification Performance via the Majority Vote Rule.

If one trains three networks with three different, but equivalent, training data sets, it is possible to use a majority vote rule to realize an improvement in average classification performance. The results showed that 30 majority vote networks, constructed from individual nets trained on slightly different data sets, averaged $1.8 \%$ better performance than 30 individual nets trained in the same manner. Statistical tests on the joint recognition PDFs showed this difference to be significant.

Influence of Training Data Sets, Initial Weights, and Exemplar Presentation Order on Network Solutions.

The influence of the training data set strongly outwcighs the influence of initial starting weights and/or the order of presentation of training exemplars, with regard to the decision regions formed by a given network. An examination of the incorrectly classified test set exemplars was performed on sets of 30 nets trained for each of four runs. In Run 2 , the exact composition of the training set, initial starting weights, and presentation order of exemplars, was different for each net trained. In Run 3 , only the initia weights and presentation order were different. In Run 4, only the initial weights were different and in Run 5 , only the presentation orders were different. The values of $L$, a metric measuring the correlation between the decision regions formed by the nets within a given run, for Runs $2,3,4$ and 5 were $0.362,0.546,0.606$, and 0.667 , respectively. These values clearly suggest a relative order and degree of impact the conditions have on the exact shape of decision regions formed. The $L$ values indicate that exact composition of the training data set is most important, the initial set of weights the next most important, and the order of exemplar presentation the least important.

\section{References}

[1] R. L. Pickholtz et al., "Theory of Spread-Spectrum Communication a Tutorial," IEEE Transactions on Communications, vol. 30 , pp. 855884, May 1982

[2] C. Garvin, Personal Correspondence. U.S. Army, Harry Diamond Laboratories, Adelphi, Maryland, January 1989.

[3] G. L. Tarr, "Dynamic Analysis of Feedforward Neural Networks Using Simulated and Measured Data," Master's thesis AFIT/GE/ENG/88D-54. School of Engineering, Air Force Institute of Technology (AU), Wright-Patterson AFB, Ohio, December 1988.

[4] R. P. Lippmann, "An Introduction to Computing with Neural Nets," IEEE ASSP Magazine, pp. 4-22, April 1987.

[5] R. W. Hamming, Coding and Information Theory. Prentice-Hall, second ed., 1986.

[6] C. C. Piazza, "Modified Backward Error Propagation for Tactical Target Recognition," Master's thesis, AFIT/GE/ENG/88D-36. School of Enginecring, Air Force Institute of Technology (AU), Wright-Patterson AFB, Ohio, December 1988.

[7] NH Analytical Software, 1958 Eldrige Avenue, Roseville MN 55113 Statistix ${ }^{i m}$, An Interactive Statistical Analysis Program for Microcomputers.

[8] S. S. Shapiro and M. B. Wilk, "An Analysis of Variance Test for Normality," Biometrika, vol. 52, pp. 591-611, 1965.

[9] S. D. Schlotzhauer and R. C. Littell, $S A S^{\text {tm }}$ System for Elementary Statistical Analysis. SAS Institute Inc., Box 8000 , SAS Circle, Cary NC $27512-8000$.

[10] I. Miller and J. E. Freund, Probability and Statistics for Engineers. Prentice-Hall, third ed., 1985.

Table 1: Summary of Experiment Run Parameters

\begin{tabular}{|c|c|c|c|c|c|c|}
\hline & & & Run & signation & & \\
\hline Parameter & R̂ 1 & RIA & $\mathrm{R} 2$ & $\mathrm{R} 3$ & $\mathrm{R} 4$ & $\mathrm{R} 5$ \\
\hline \begin{tabular}{|l} 
\# of Nets \\
Trained
\end{tabular} & 30 & - & 120 & 120 & 120 & 120 \\
\hline $\begin{array}{l}\text { \# of Traising } \\
\text { Exemplars } \\
\end{array}$ & 102 & - & 85 & 102 & 502 & 102 \\
\hline $\begin{array}{l}\text { Training } \\
\text { Set Mix } \\
\text { class } 1 / \text { class } 2\end{array}$ & $50 / 50 \%$ & * & $60 / 40 \%$ & $50 / 50 \%$ & $50 / 50 \%$ & $50 / 50 \%$ \\
\hline $\begin{array}{l}\text { Identical } \\
\text { Training, Sets } \\
\end{array}$ & Yes & • & No & Yes & Yes & Yes \\
\hline $\begin{array}{l}\text { \# of Test } \\
\text { Exemplars }\end{array}$ & 100 & 95 & 100 & 100 & 100 & 100 \\
\hline $\begin{array}{l}\text { Test Set Mix } \\
\text { class } \mathrm{1} / \text { class } 2\end{array}$ & $50 / 50 \%$ & $40 / 60 \%$ & $50 / 50 \%$ & $50 / 50 \%$ & $50 / 50 \%$ & $50 / 50 \%$ \\
\hline $\begin{array}{l}\text { Adentical } \\
\text { Test Sets } \\
\end{array}$ & Yes & No & Yes & Yes & Yes & Yes \\
\hline $\begin{array}{l}\text { Generator Seed } \\
\text { for Initial } \\
\text { Weights }\end{array}$ & Variatle & Variable & Variable & Variable & Variable & Fixed \\
\hline $\begin{array}{l}\text { Generator Seed } \\
\text { for Presentation } \\
\text { Order }\end{array}$ & Variable & Variable & Variable & Variable & Fixed & Variable \\
\hline $\begin{array}{l}\text { Majority Voie } \\
\text { Networks }\end{array}$ & No & No & Yes & Yes & Yes & Yes \\
\hline $\begin{array}{l}\text { Distribu } \\
\text { Nomencl }\end{array}$ & \begin{tabular}{|c|}
$R 1 P 11$ \\
R1P22 \\
R1PG \\
CR1PG
\end{tabular} & $\begin{array}{l}\text { R1APII } \\
\text { R1AP22 } \\
\text { R1APG }\end{array}$ & \begin{tabular}{|c|} 
R2SP11 \\
R2SP22 \\
R2SPG \\
R2MP11 \\
R2MP22 \\
R2MPG \\
CR2MP11 \\
CR2MP22 \\
CR2MPG \\
\end{tabular} & $\begin{array}{l}\text { R3SP11 } \\
\text { R3SP22 } \\
\text { R33PG } \\
\text { R3MP11 } \\
\text { R3MP22 } \\
\text { R3MPG } \\
\text { CRMMP11 } \\
\text { CR3MP22 } \\
\text { CR3MPG }\end{array}$ & $\begin{array}{l}\text { R4SP11 } \\
\text { RASP22 } \\
\text { R4SPG } \\
\text { R4MP11 } \\
\text { R4MP22 } \\
\text { RAMPG } \\
\text { CRMMP11 } \\
\text { CRAMP22 } \\
\text { CR4MPG } \\
\end{array}$ & \begin{tabular}{|l} 
R5SPI1 \\
R5SP22 \\
R5SPG \\
R5MP11 \\
R5MP22 \\
R5MPG \\
CR5MP11 \\
CR5MP22 \\
CR5MPG
\end{tabular} \\
\hline
\end{tabular}




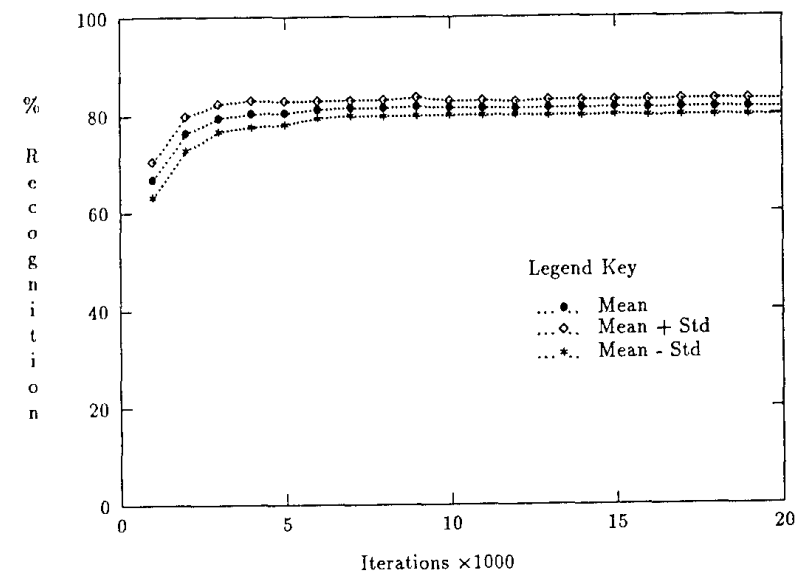

Figure 1: Training Histories of Good Classification on Test Data for Run 3

Table 3: Results of Ilypothesis Tests Between Run 1 and Run 1a Distributions

\begin{tabular}{|c|c|c|c|c|c|c|c|c|}
\hline \multicolumn{9}{|c|}{$\begin{array}{l}H_{0} \text { : Samples are from the same distribution } \\
\text { Criterion: Reject null if } p<0.05 \text {, otherwise. }\end{array}$} \\
\hline Test & \multicolumn{2}{|c|}{$\begin{array}{c}\text { Wilcoxon } \\
\text { Signed Rank }\end{array}$} & \multicolumn{2}{|c|}{$\begin{array}{l}\text { Rank } \\
\text { Sum }\end{array}$} & \multicolumn{2}{|c|}{$\begin{array}{l}\text { Kruskal-Wallis } \\
\text { One Way AOV }\end{array}$} & \multicolumn{2}{|c|}{$\begin{array}{c}\text { Median } \\
\text { Test }\end{array}$} \\
\hline Comparison & $p$ & $F / R$ & $p$ & $\overrightarrow{\mathrm{F}} / \mathrm{R}$ & $p$ & $F / R$ & $p$ & $\mathrm{~F} / \overline{\mathrm{R}}$ \\
\hline R1P11-R1AP1I & .1156 & $\mathrm{~F}$ & .4643 & $\mathrm{~F}$ & .4577 & $\mathrm{~F}$ & .2274 & $\bar{F}$ \\
\hline$R_{1 P 22}-R 1 A P 22$ & .0000 & $\mathrm{R}$ & .0215 & $\mathrm{R}$ & .0186 & $\mathrm{R}$ & .0503 & $\mathrm{~F}$ \\
\hline$R \perp P G-R I A P G$ & .0000 & $\mathrm{R}$ & .0001 & $\mathrm{R}$ & .0001 & $\mathrm{R}$ & .0001 & $\mathrm{R}$ \\
\hline$C R \perp P G-R I A P G$ & .6343 & $\mathrm{~F}$ & .7731 & $\mathrm{~F}$ & .7668 & $\mathrm{~F}$ & .8172 & $\mathrm{~F}$ \\
\hline
\end{tabular}

Table 5: Results of Hypothesis Tests Between Run 1 and Run 2S Distributions

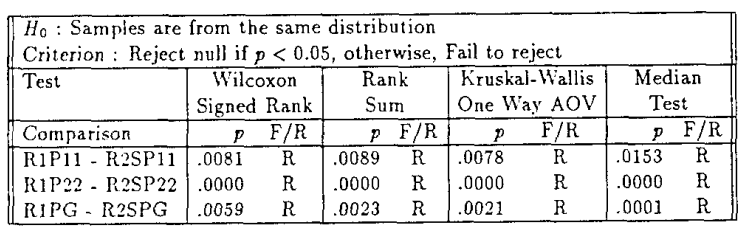

Table 2: Summary Statistics for Distributions of Run 1 and Run 1A

\begin{tabular}{||c|c|c|c|c|c||}
\hline Run & \multirow{2}{*}{ ID } & Metric & $P(1 \mid 1)$ & $P(2 \mid 2)$ & \multicolumn{2}{|c|}{$P$ (good) } \\
\cline { 5 - 6 } & & & Observed & Calculated \\
\hline R1 & Mean & 0.7380 & 0.9213 & 0.8297 & 0.8480 \\
& STD & 0.0384 & 0.0185 & 0.0190 & 0.0164 \\
\hline R1A & Mean & 0.7263 & 0.9304 & 0.8488 & NA \\
& STD & 0.0542 & 0.0178 & 0.0223 & NA \\
\hline \multirow{2}{*}{$\begin{array}{c}\text { Difference } \\
\text { of Means }\end{array}$} & \multicolumn{3}{|c|}{ R1A - R1 } & R1A - CR1 \\
\cline { 2 - 5 } & -0.0117 & 0.0091 & 0.0191 & .0008 \\
\hline
\end{tabular}

Table 4: Summary Statistics for Distributions of Run 1 and Run 2S

\begin{tabular}{||c|c|c|c|c||}
\hline $\begin{array}{c}\text { Run } \\
\text { ID }\end{array}$ & Metric & $P(1 \mid 1)$ & $P(2 \mid 2)$ & $P($ good) \\
\hline R1 & Mean & 0.7380 & 0.9213 & 0.8297 \\
& STD & 0.0384 & 0.0185 & 0.0190 \\
\hline R2S & Mean & 0.7767 & 0.8320 & 0.8043 \\
& STD & 0.0485 & 0.0560 & 0.0374 \\
\hline \multicolumn{2}{|c|}{$\begin{array}{c}\text { Difference } \\
\text { of Means }\end{array}$} & \multicolumn{4}{|c|}{ R2S - R1 } \\
\cline { 2 - 5 } & & & &
\end{tabular}

Table 6: Summary of Means for $P$ (good) Distributions of Run 2 through Run 5

\begin{tabular}{||c|c|c|c|c|c||}
\hline \multirow{2}{*}{ Run } & \multicolumn{3}{|c|}{ PDF ID } & \multicolumn{2}{|c||}{ Diflerences } \\
\cline { 2 - 6 } & $\mathrm{S}$ & $\mathrm{M}$ & $\mathrm{CM}$ & $\mathrm{M}-\mathrm{S}$ & $\mathrm{CM}-\mathrm{S}$ \\
\hline R2 & 0.8043 & $\mathbf{0 . 8 2 2 3}$ & 0.8957 & 0.0180 & 0.0914 \\
R3 & 0.8140 & 0.8200 & 0.8998 & 0.0060 & 0.0858 \\
R4 & 0.8183 & 0.8190 & 0.8910 & 0.0007 & $\mathbf{0 . 0 7 2 7}$ \\
R5 & 0.8103 & 0.8083 & 0.8863 & -0.0020 & 0.0760 \\
\hline
\end{tabular}

Table 7: Results of Hypothesis Tests Between PDFs of Single and Majority Vote Nets for Run 2 through Run 5

\begin{tabular}{|c|c|c|c|c|c|c|c|c|c|c|}
\hline \multicolumn{11}{|c|}{$\begin{array}{l}\text { Ho: Samples are from the same distribution } \\
\text { Criterion : Reject null if } p<0.05 \text {, otherwise, Fail to reject }\end{array}$} \\
\hline Test & \multicolumn{2}{|c|}{$\begin{array}{c}\text { Wilcoxon } \\
\text { Signed } \\
\text { Rank }\end{array}$} & \multicolumn{2}{|c|}{$\begin{array}{l}\text { Rank } \\
\text { Sum }\end{array}$} & \multicolumn{2}{|c|}{$\begin{array}{l}\text { Kruskal- } \\
\text { Wallis One } \\
\text { Way AOV }\end{array}$} & \multicolumn{2}{|c|}{$\begin{array}{c}\text { Median } \\
\text { Test }\end{array}$} & \multicolumn{2}{|c|}{$\begin{array}{c}\text { Two } \\
\text { Sample } \\
\text { T Test }\end{array}$} \\
\hline Comparison & $p$ & $F / R$ & $p$ & $F / R$ & $p$ & $F / R$ & $p$ & $\mathrm{~F} / \mathrm{R}$ & $p$ & $\mathrm{~F} / \mathrm{R}$ \\
\hline R2SPG - R2M $\overline{P G}$ & .0490 & $\mathrm{R}$ & .0315 & $\mathrm{R}$ & .0297 & $\overline{\mathrm{R}}$ & .0175 & $\mathrm{R}$ & 0.0249 & $\mathrm{R}$ \\
\hline$R 3 S P G-R 3 M P G$ & .1886 & $F$ & .2009 & $\mathrm{~F}$ & .1897 & $F$ & $.167 \pi$ & $F$ & 0.1789 & F \\
\hline R4SPG - R4MPG & .7897 & $\mathrm{~F}$ & .9058 & $\mathrm{~F}$ & .8983 & $\mathrm{~F}$ & .8841 & $\mathrm{~F}$ & $\mathrm{NA}$ & $\mathrm{NA}$ \\
\hline R5SPG - R5MPG & .5531 & $\mathrm{~F}$ & .6361 & $\mathrm{~F}$ & .6207 & $\mathrm{~F}$ & .3659 & $\mathrm{~F}$ & NA & $\mathrm{NA}$ \\
\hline
\end{tabular}


Table 8: Incorrectly Classified Test Exemplars for Run 2 Through Run 5

\begin{tabular}{|c|c|c|c|c|c|c|c|}
\hline \multicolumn{2}{|c|}{ Fun 25} & \multicolumn{2}{|c|}{ Run 35} & \multicolumn{2}{|c|}{ Kun 4S } & \multicolumn{2}{|c|}{ Run $\overline{5 S}$} \\
\hline File & Count & File & Count & File & Count & File & Count \\
\hline corr96 & 30 & corrli & 30 & corr17 & 30 & corr25 & 30 \\
\hline corr 25 & 29 & corr 25 & 30 & corr 25 & 30 & corr 37 & 30 \\
\hline corr194 & 28 & corr 37 & 30 & corr 37 & 30 & сот'? & 30 \\
\hline corr53 & 27 & corr 96 & 30 & corral & 30 & corrs1 & 30 \\
\hline corr 227 & 27 & corr227 & 30 & corr 96 & 30 & corr 164 & 30 \\
\hline corrl64 & 26 & corr194 & 30 & corr194 & 30 & corr 182 & 30 \\
\hline corr 7 & 24 & corr 53 & 29 & $\operatorname{corr} 168$ & 29 & corr194 & 30 \\
\hline corr182 & 24 & corr 7 & 28 & corr53 & 28 & corr29 & 29 \\
\hline corr 37 & 23 & corrs I & 28 & corr164 & 28 & corr53 & 29 \\
\hline corr 145 & 23 & corr164 & 28 & corr182 & 28 & corrlito & 29 \\
\hline corr170 & 22 & corr182 & 28 & corr 227 & 28 & $\operatorname{corr} 241$ & 29 \\
\hline corr168 & 20 & corr 168 & 26 & $\operatorname{corr} 7$ & 27 & corr17 & 27 \\
\hline corrit & 17 & corr169 & 26 & corr 19 & 26 & corr19 & 27 \\
\hline corr92 & 16 & corr145 & 25 & corr 170 & 25 & corr145 & 27 \\
\hline corr 241 & 16 & corr41 & 24 & corr 51 & 23 & corr22i & 26 \\
\hline corrs2 & 15 & corr241 & 21 & corr152 & 22 & corr $\bar{t}$ & 22 \\
\hline corris & 13 & corr29 & 20 & corr176 & 15 & corrst & 21 \\
\hline corr 31 & 12 & corr19 & 19 & corr241 & 15 & corr 41 & 19 \\
\hline corr 57 & 12 & corr 57 & 17 & corr 29 & $\begin{array}{l}14 \\
14\end{array}$ & corr168 & 19 \\
\hline corr 90 & 11 & corr82 & 12 & corrs2 & 10 & corr 176 & 19 \\
\hline corr 41 & 10 & corre35 & 9 & $\operatorname{corr} 200$ & 10 & corrs? & 13 \\
\hline corroso & 10 & corr 39 & 7 & corr57 & 9 & corr200 & 7 \\
\hline corris & 10 & corr176 & 7 & corr 31 & 8 & corr31 & 4 \\
\hline $\operatorname{corr} 88$ & 10 & corr 205 & 4 & corr 35 & 5 & corr39 & 4 \\
\hline corr 64 & & corr 200 & 4 & corrso & 3 & $\operatorname{corr} 151$ & 3 \\
\hline corr66 & 9 & corr31 & 3 & $\operatorname{cotr} 39$ & 3 & corr 35 & 2 \\
\hline corr 51 & 9 & $\operatorname{corr} 128$ & 3 & corr 43 & 3 & $\operatorname{corr} 45$ & 1 \\
\hline corr35 & 8 & corr27 & 2 & corr 190 & 3 & corr219 & 1 \\
\hline $\begin{array}{l}\text { corr84 } \\
\text { corr62 }\end{array}$ & $\begin{array}{l}8 \\
7\end{array}$ & $\begin{array}{l}\text { corr1190 } \\
\text { corr13 }\end{array}$ & $\begin{array}{l}2 \\
1\end{array}$ & $\begin{array}{l}\text { corr27 } \\
\text { corr184 }\end{array}$ & $\begin{array}{l}2 \\
1\end{array}$ & $\begin{array}{l}\text { corr } 190 \\
\text { corr202 }\end{array}$ & 1 \\
\hline corrio & 7 & corr 43 & 1 & & & & \\
\hline $\begin{array}{l}\text { corr } 254 \\
\text { corr } 29\end{array}$ & $\frac{7}{7}$ & $\begin{array}{l}\text { corr59 } \\
\text { corr178 }\end{array}$ & $\begin{array}{l}1 \\
1\end{array}$ & & & & \\
\hline corr 27 & 6 & corr201 & 1 & & & & \\
\hline $\begin{array}{l}\text { corr 176 } \\
\text { corr } 243\end{array}$ & $\begin{array}{l}5 \\
5\end{array}$ & & & & & & \\
\hline $\begin{array}{l}\text { corr } 243 \\
\text { corr68 }\end{array}$ & 4 & & & & & & \\
\hline corr72 & 4 & & & & & & \\
\hline $\begin{array}{l}\operatorname{corr} 190 \\
\operatorname{corr} 205\end{array}$ & $\begin{array}{l}4 \\
4\end{array}$ & & & & & & \\
\hline $\operatorname{corr} 233$ & 4 & & & & & & \\
\hline $\begin{array}{l}\operatorname{corr} 148 \\
\operatorname{corr} 200\end{array}$ & $\begin{array}{l}3 \\
3\end{array}$ & & & & & & \\
\hline corr 202 & 3 & & & & & & \\
\hline $\operatorname{corr} 231$ & 3 & & & & & & \\
\hline $\begin{array}{l}\operatorname{cotr} 235 \\
\text { corr } 43\end{array}$ & $\begin{array}{l}3 \\
2 \\
2\end{array}$ & & & & & & \\
\hline corr147 & 2 & & & & & & \\
\hline corr39 & $\begin{array}{l}1 \\
1\end{array}$ & & & & & & \\
\hline $\begin{array}{l}\text { corr151 } \\
\text { corr211 }\end{array}$ & 1 & & & & & & \\
\hline corr213 & 3 & & & & & & \\
\hline $\begin{array}{l}\text { corr223 } \\
\text { corr225 }\end{array}$ & $\begin{array}{l}1 \\
1\end{array}$ & & & & & & \\
\hline
\end{tabular}

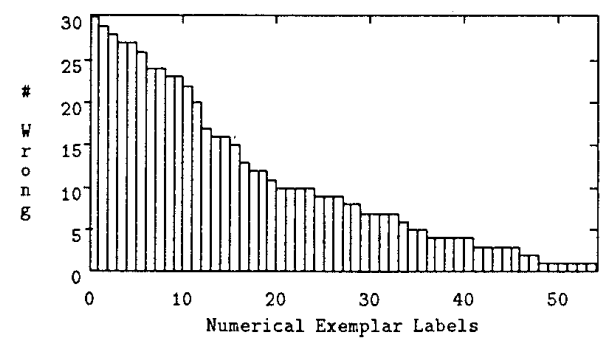

Figure 2: Run 2 Incorrectly Classified Exemplar Counts

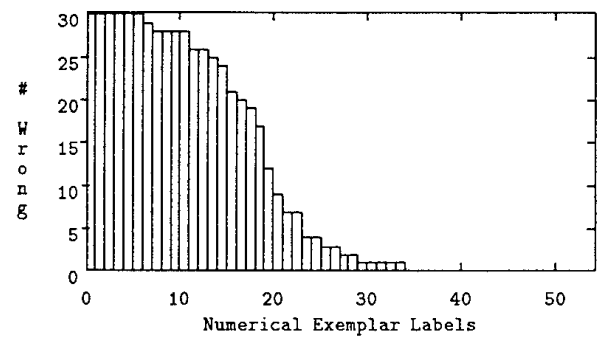

Figure 3: Run 3 Incorrectly Classified Exemplar Counts

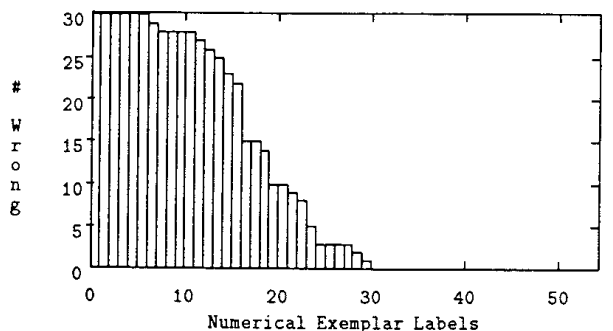

Figure 4: Run 4 Incorrectly Classified Exemplar Counts

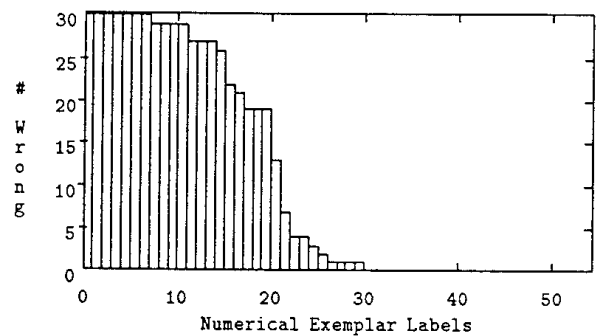

Figure 5: Run 5 Incorrectly Classified Exemplar Counts 